\title{
On the existence theorems of solutions for generalized vector variational inequalities
}

\author{
Shih-sen Chang ${ }^{1 *}$, Salahuddin² and Gang Wang ${ }^{3}$
}

\author{
"Correspondence: \\ changss2013@163.com \\ 'Center for General Education, \\ China Medical University, Taichung, \\ 40402, Taiwan \\ Full list of author information is \\ available at the end of the article
}

\begin{abstract}
In this paper, we prove some existence theorems of solutions for two classes of generalized vector variational inequalities and Minty generalized vector variational inequalities.
\end{abstract}

MSC: 46A50; 47H04

Keywords: generalized vector variational inequalities; Minty generalized vector variational inequality; cones; quasilinear type operator; Minty lemma; KKM mapping

\section{Introduction}

The variational inequality theory, which is mainly due to Stampacchia [1], provides very powerful techniques for studying problems arising in mechanics, optimization, transportation, economics, contact problems in elasticity, and other branches of mathematics. The free boundary value problem can be studied effectively in the framework of variational inequalities, the traffic assignment problem is a variational inequality problems. The theory of vector variational inequalities was initiated by Giannessi [2]. The theory has shown to be very useful in studying problems arising in pure and applied sciences, engineering and technology, financial mathematics, transportation, and other problems of practical interest, see [3-10]. In recent years, a considerable number of generalizations of vector variational inequalities have been considered, studied, and applied in various directions. The general variational inequality problems provide us with a unified, simple, innovative, and natural frame work for studying a wide class of problems involving unilateral, moving boundary, obstacle, free boundary and equilibrium problems. The existence problems of solutions for this problems are helpful in the sense that one would like to know if a solution of a general variational inequality exists before one actually devises some plausible algorithms for solving the problems; while existence results of the solution for Stampacchia variational inequalities were abundant in the last years, this is not the case of general variational inequalities of Stampacchia type [1].

Inspired and motivated by the recent work [11-21], the purpose of this paper is first to study a new class of quasilinear type operators and then to establish some existence theorems of solutions for a class of generalized vector variational inequalities and Minty generalized vector variational inequalities.

(c) 2015 Chang et al. This article is distributed under the terms of the Creative Commons Attribution 4.0 International License (http://creativecommons.org/licenses/by/4.0/), which permits unrestricted use, distribution, and reproduction in any medium, provided you give appropriate credit to the original author(s) and the source, provide a link to the Creative Commons license, and indicate if changes were made. 


\section{Preliminaries}

In what follows, unless otherwise specified, we assume that $X$ is a Banach space and $X^{*}$ is its topological dual. We denote by $\left\langle x^{*}, x\right\rangle$ the value of the linear and continuous functional $x^{*} \in X^{*}$ in $x \in X$. Let $K$ be a nonempty closed convex subset of a Banach space $X, A$ : $K \subset X \rightarrow X^{*}$ and $a: K \rightarrow X$ be two given operators. Let $C: K \rightarrow 2^{X}$ be a mapping with closed convex cone values such that for each $x \in K$, int $C(x) \neq \emptyset$ and satisfying the following conditions:

(i) $\lambda C(x) \subset C(x)$, for $\lambda>0$;

(ii) $C(x)+C(x) \subset C(x)$;

(iii) $C(x) \cap(-C(x))=\{0\}$.

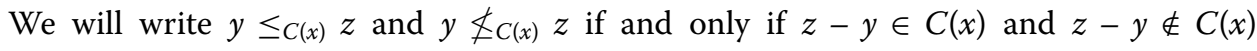
for all $x, y, z \in K$, respectively. We will write $y \leq \operatorname{int} C(x) z$ if $z-y \in \operatorname{int} C(x)$ in the case $\operatorname{int} C(x) \neq \emptyset$.

The problem that we shall study in this paper is the so-called generalized vector variational inequalities $\operatorname{GVVI}(A, a, K)$ i.e., to find a $x \in K$ such that

$$
\langle A(x), a(y)-a(x)\rangle \notin-\operatorname{int} C(x), \quad \forall y \in K .
$$

We note that if $a \equiv \mathrm{id}_{K}$, then (1) reduces to the problem for finding $x \in K$ such that

$$
\langle A(x), y-x\rangle \notin-\operatorname{int} C(x), \quad \forall y \in K,
$$

which is called general vector variational inequalities $\operatorname{GVVI}(A, K)$ studied by [2].

The so-called Minty generalized vector variational inequality $\operatorname{MGVVI}(A, a, K)$ is to find a $x \in K$ such that

$$
\langle A(y), a(y)-a(x)\rangle \notin-\operatorname{int} C(x), \quad \forall y \in K
$$

Note that if $a \equiv \operatorname{id}_{K}$ then (3) reduces to the Minty general vector variational inequality $\operatorname{MGVVI}(A, K)$ for finding $x \in K$ such that

$$
A(y), y-x\rangle \notin-\operatorname{int} C(x), \quad \forall y \in K,
$$

where $K$ is a convex and closed set.

Let $X$ be a real linear space. For $x, y \in X$ we denote by $[x, y]=\{(1-t) x+t y: t \in[0,1]\}$ the closed line segment with the end point $x$ and $y$. The open line segment with endpoints $x$, respectively $y$, is defined by $(x, y)=[x, y] \backslash\{x, y\}=\{z=(1-t) x+t y: t \in(0,1)\}$. Let $Y$ be another real linear space. An operator $A: D \subseteq X \rightarrow Y$ is said to be quasilinear if

$$
A([x, y] \cap D) \subseteq[A(x), A(y)], \quad \text { for every } x, y \in D .
$$

Theorem 2.1 [22] Let $f: I \subseteq R \rightarrow R$ be a function. Then $f$ is a quasilinear if and only iff is monotone.

Theorem 2.2 [22] Let $A: X \rightarrow Y$ be a linear operator. Then $A$ is quasilinear. 
Theorem 2.3 [22] Let $D \subseteq X$ be a convex set and $A: D \rightarrow Y$ be a quasilinear operator. Then for every $n \in N$ and every $x_{1}, \ldots, x_{n} \in D$ and every $x \in \operatorname{co}\left\{x_{1}, x_{2}, \ldots, x_{n}\right\}$ we have

$$
A(x) \in \operatorname{co}\left\{A\left(x_{1}\right), \ldots, A\left(x_{n}\right)\right\}
$$

where $\operatorname{co}(E)$ denotes the convex hull of the set $E \subseteq Y$.

Definition 2.4 Let $X$ and $Y$ be two real linear spaces. Then the operator $A: D \subseteq X \rightarrow Y$ is quasilinear if for every $x, y \in D$ and every $z \in[x, y] \cap D$,

$$
A(z) \in[A(x), A(y)]
$$

Proposition 2.5 Let $X$ and $Y$ be two real linear spaces and $A: D \subseteq X \rightarrow Y$ be a quasilinear operator. Then $\lambda A: D \rightarrow Y$ is quasilinear for all $\lambda \in R$.

Proof For $x, y \in D, z \in[x, y] \cap D$ it follows from Definition 2.4 that

$$
A(z) \in[A(x), A(y)]
$$

Therefore

$$
\lambda A(z) \in[\lambda A(x), \lambda A(y)] .
$$

Definition 2.6 Let $U$ and $V$ be two real linear spaces and $A: D \subseteq U \rightarrow V$ be an operator. $A$ is said to be $c$-monotone, if for every $v \in V$ the set $A^{-1}(v)$ is convex.

Proposition 2.7 Let $X$ and $Y$ be two real linear spaces, $D \subseteq X$ be a convex set and $A: D \rightarrow$ $Y$ be a quasilinear mapping. Then $A$ is c-monotone.

Proof Suppose to contrary that $A$ is not $c$-monotone. Then there exists $u \in Y$ such that $A^{-1}(u)$ is not convex. Consequently for $x, y \in A^{-1}(u), x \neq y$ there exists $z \in(x, y)$ with $A(z) \neq u$. Since $A$ is quasilinear,

$$
A(z) \in[A(x), A(y)]=\{u\}
$$

a contradiction.

Definition 2.8 Let $D$ be an open subset of a topological space $X$ and $f: D \rightarrow Y$ be an operator where $Y$ is an arbitrary set. Then $f$ is said to be locally injective, if for each $x \in D$, it admits a neighborhood $U_{x} \subseteq D$ such that $f$ is injective on $U_{x}$ i.e., $f(u) \neq f(v)$ for all $u, v \in U_{x}$, $u \neq v$.

Proposition 2.9 Let $X$ be a topological real linear space, $Y$ be a real linear space and $D \subseteq X$ be a convex and open subset. Let $A: D \rightarrow Y$ be quasilinear and locally injective. Then $A$ is injective.

Proof Now we prove that for each $u \in A(D), A^{-1}(u)$ contains only one point. Assume that there exist $x, y \in A^{-1}(u), x \neq y$. Since $A$ is locally injective, there exists a neighborhood $U_{x}$ 
of $x$ contained in $D$ such that $A(z) \neq A(x)$ for $z \neq x, z \in U_{x}$. Since $A$ is $c$-monotone and $[x, y] \subseteq A^{-1}(u), z \in U_{x} \cap(x, y]$. Hence $A(z)=u$ and $A(z) \neq A(x)=u$, a contradiction.

Definition 2.10 Let $X$ be a real linear spaces and $Y$ be a topological space. Let $A: D \subseteq$ $X \rightarrow Y$ be an operator. We say $A$ is continuous on line segment at $x \in D$ if for every sequence $\left\{t_{n}\right\} \subseteq R$ of a real number convergent to 0 and every $x, y \in D$ with $x+t_{n} y \in D$ we have

$$
A\left(x+t_{n} y\right) \rightarrow A(x), \quad n \rightarrow \infty .
$$

We say that $A$ is continuous on line segments in $D$ if it has the continuity property at every $x \in D$.

Lemma 2.11 [23] Let $X$ be a real linear space. Let $Y$ be a real linear metric space and $D \subseteq X$ be a convex subset. Let $A: D \rightarrow Y$ be a quasilinear operator which is continuous on line segments. Then for every $x, y \in D$ we have

$$
A([x, y])=[A(x), A(y)] .
$$

Remark 2.12 In Lemma 2.11, if $A$ is also injective, then for every $x, y \in D, x \neq y$ we have

$$
A((x, y))=(A(x), A(y)) .
$$

Proposition 2.13 Let $X$ be a real linear space, $Y$ be a real linear metric space. Let A : $D \subseteq X \rightarrow Y$ be an injective convex and quasilinear mapping and it is continuous on line segments. Then the operator $A^{-1}: A(D) \rightarrow D$ is quasilinear.

Proof Since $A$ is injective, $A^{-1}: A(D) \rightarrow D$ is single valued. Suppose there exist $u, v \in A(D)$ and $w \in(u, v)$ such that

$$
A^{-1}(w) \notin\left(A^{-1}(u), A^{-1}(v)\right) .
$$

Denote by $x=A^{-1}(u), y=A^{-1}(v), z=A^{-1}(w)$ then from Remark 2.12 we have $A(x, y)=$ $(A(x), A(y))$ and there exists $z^{\prime} \in(x, y)$ such that $A\left(z^{\prime}\right)=A(z)=w$. From the injectivity of $A$ we have $z=z^{\prime}$, which is a contradiction. Therefore $z \notin(x, y)$.

Theorem 2.14 Let $X$ be a real linear space, $Y$ be a real linear space and also metric space. Let $A: D \subseteq X \rightarrow Y$ be continuous on line segments, quasilinear with its domain $D$ and convex. Then $A(D)$ is convex.

Proof Let $u, v \in A(D)$, then we prove that $[u, v] \subseteq A(D)$. Let $x \in A^{-1}(u), y \in A^{-1}(v)$, from Lemma $2.11 A([x, y])=[A(x), A(y)]$, therefore we have

$$
[u, v]=A([x, y]) \subseteq A(D) .
$$

Definition 2.15 Let $X$ be a real linear space and $D \subseteq X$ be convex. A function $f: D \rightarrow R$ is called quasiconvex if for every $x, y \in D$ and $t \in[0,1]$ we have

$$
f((1-t) x+t y) \leq \max \{f(x), f(y)\} .
$$


$f$ is called quasiconcave [24] if $-f$ is quasiconvex i.e.,

$$
f((1-t) x+t y) \geq \min \{f(x), f(y)\}, \quad \forall x, y \in D, t \in[0,1] .
$$

A function that is quasiconvex and quasiconcave at the same time is called quasilinear.

Proposition 2.16 Let $X, Y, Z$ be real linear spaces, and let $D \subseteq X$ and $A: D \rightarrow Y, B$ : $A(D) \rightarrow Z$ be two quasilinear operators. Then $B \circ A: D \rightarrow Z$ is quasilinear.

Proof For $x, y \in D$ we have $A([x, y] \cap D) \subseteq[A(x), A(y)]$. For $u, v \in A(D)$ we have $B[u, v] \cap$ $A(D) \subseteq[B u, B v]$. Let $z \in[x, y] \cap D$ and $w=A(z), u=A(x), v=A(y)$. Therefore, $w \in[u, v]$ and $B$ is quasilinear, and we get $B(w) \in[B(u), B(v)]$, that is,

$$
(B \circ A)(z) \in[(B \circ A)(x),(B \circ A)(y)] .
$$

Definition 2.17 Let $X$ be a real linear space and $D \subseteq X$. The convex hull of the set $D$ is defined by

$$
\operatorname{co}(D)=\left\{\sum_{i=1}^{n} \lambda_{i} x_{i}: x_{i} \in D, \sum_{i=1}^{n} \lambda_{i}=1, \lambda_{i} \geq 0, \forall i \in\{1,2, \ldots, n\}, n \in N\right\} .
$$

Theorem 2.18 Let $X$ and $Y$ be two real linear spaces, and $D \subseteq X$ be a convex subset. Let $A: D \rightarrow Y$ be quasilinear, then for every $n \in N$, every $x_{1}, x_{2}, \ldots, x_{n} \in D$ and every $x \in$ $\operatorname{co}\left\{x_{1}, x_{2}, \ldots, x_{n}\right\}$, we have

$$
A(x) \in \operatorname{co}\left\{A\left(x_{1}\right), A\left(x_{2}\right), \ldots, A\left(x_{n}\right)\right\} .
$$

Theorem 2.19 Let $Y$ be a topological vector spaces with a pointed closed and convex cone $C$ such that int $C \neq \emptyset$, then for all $x, y, z \in Y$ we have

(i) $x-y \in-\operatorname{int} C$ and $x \notin-\operatorname{int} C \Rightarrow y \notin-\operatorname{int} C$;

(ii) $x+y \in-C$ and $x+z \notin-\operatorname{int} C \Rightarrow z-y \notin-\operatorname{int} C$;

(iii) $x+z-y \notin-\operatorname{int} C$ and $-y \in-C \Rightarrow x+z \notin-\operatorname{int} C$;

(iv) $x+y \notin-\operatorname{int} C$ and $y-z \in-C \Rightarrow x+z \notin-\operatorname{int} C$.

\section{Existence of solutions for generalized vector variational inequalities}

In this section we present some existence results of solutions for vector variational inequalities (1) and (3).

Definition 3.1 (Knaster-Kuratowski-Mazurkiewicz) Let $X$ be a Hausdorff linear space and $M \subseteq X$. The mapping $G: M \rightarrow 2^{X}$ is called a KKM mapping, if for each finite number of elements $x_{1}, x_{2}, \ldots, x_{n} \in M$ we have

$$
\operatorname{co}\left\{x_{1}, \ldots, x_{n}\right\} \subseteq \bigcup_{j=1}^{n} G\left(x_{i}\right)
$$

Lemma 3.2 [25] Let $X$ be a Hausdorff linear space, $M \subseteq X$ and $G: M \rightarrow 2^{X}$ be a KKM mapping. If $G(x)$ is closed for every $x \in M$ and there exists $x_{0} \in M$ such that $G\left(x_{0}\right)$ is compact 
then

$$
\bigcap_{x \in M} G(x) \neq \emptyset
$$

An operator $T: X \rightarrow X^{*}$ is called weak to $\|\cdot\|$-sequentially continuous at $x \in X$ if, for every sequence $\left\{x_{n}\right\}$ which converges weakly to $x$, we have $\left\{T\left(x_{n}\right)\right\} \rightarrow T(x)$ in the topology of the norm of $X^{*}$. An operator $T: X \rightarrow X$ is called weak to weak sequentially continuous at $x \in X$ if, for every sequence $\left\{x_{n}\right\}$ which converges weakly to $x$, we find that $\left\{T\left(x_{n}\right)\right\}$ converges to $T(x)$.

Lemma 3.3 If $P \subset Q \subset X$ where $Q$ is weakly compact and $P$ is weakly sequentially closed, then $P$ is weakly compact.

Proof From the Eberlein-Smulian theorem [26], $Q$ is weakly sequentially compact. Let $\left\{x_{k}\right\} \subseteq P$, hence $\left\{x_{k}\right\} \subseteq Q$, which is weakly sequentially compact. Hence there exists $\left\{x_{k_{n}}\right\} \subseteq$ $\left\{x_{k}\right\}$, weakly converges to a point $x \in Q$. But $\left\{x_{k_{n}}\right\} \subseteq P$, which is weakly sequentially closed, hence $x \in P$. Thus $P$ is weakly sequentially compact. Therefore from the Eberlein-Smulian theorem $P$ is weakly compact.

Lemma 3.4 Let $\left\{\left(x_{i}, x_{i}^{*}\right)\right\}_{i \in I} \subset X \times X^{*}$ be a bounded net and assume that one of the following conditions is fulfilled:

(a) $\left\{x_{i}\right\}_{i \in I}$ converges to $x$ in the weak topology of $X$ and $\left\{x_{i}^{*}\right\}_{i \in I}$ converges to $x^{*}$ in the topology of norm of $X^{*}$.

(b) $\left\{x_{i}\right\}_{i \in I}$ converges to $x$ in the topology of norm of $X$ and $\left\{x_{i}^{*}\right\}_{i \in I}$ converges to $x^{*}$ in the weak ${ }^{*}$ topology of $X^{*}$.

Then $\left\langle x_{i}^{*}, x_{i}\right\rangle \rightarrow\left\langle x^{*}, x\right\rangle$.

Now we are ready to state our first main result.

Theorem 3.5 Let $X$ be a real Banach space and $X^{*}$ be its topological dual. Let $K$ be a weakly compact convex subset of $X, A: K \rightarrow X^{*}$, and $a: K \rightarrow X$ be two given operators. Assume that $C: K \rightarrow 2^{X}$ is a mapping with closed convex solid cone values and for each $x \in K$, int $C(x) \neq \emptyset$. If $A$ is weak to $\|\cdot\|$-sequentially continuous, $a$ is quasilinear and weak to weak sequentially continuous. Then the generalized vector variational inequality (1) admits a solution.

Proof Define a mapping $G: K \rightarrow 2^{K}$ by

$$
G(y)=\{x \in K:\{A(x), a(y)-a(x)\rangle \notin-\operatorname{int} C(x)\}, \quad \forall y \in K .
$$

It is easy to see that the existence of solutions of the generalized vector variational inequality (1) is equivalent to finding an element

$$
x \in \bigcap_{y \in K} G(y) .
$$

Now we first prove that $G$ is a KKM mapping with weakly compact value and all conditions in Ky Fan's lemma are satisfied. In fact, $G(y) \neq \emptyset$ and for all $y \in K$ we have $y \in G(y)$. For 
any $y \in K$, consider a sequence $\left\{x_{k}\right\} \subseteq G(y)$ converges weakly to $x \in K$. We prove that $x \in G(y)$.

Indeed we have

$$
\left\langle A\left(x_{k}\right), a(y)-a\left(x_{k}\right)\right\rangle \notin-\operatorname{int} C(x)
$$

and

$$
\begin{aligned}
\left\langle A\left(x_{k}\right), a(y)-a\left(x_{k}\right)\right\rangle= & \left\langle A\left(x_{k}\right)-A(x), a(y)-a\left(x_{k}\right)\right\rangle \\
& +\left\langle A(x), a(y)-a\left(x_{k}\right)\right\rangle \notin-\operatorname{int} C(x), \quad \forall y \in K .
\end{aligned}
$$

We prove that

$$
\left\langle A\left(x_{k}\right)-A(x), a(y)-a\left(x_{k}\right)\right\rangle \rightarrow 0, \quad k \rightarrow \infty .
$$

Indeed, from the triangle inequality, for all $y \in K$, we have

$$
\left|\left\langle A\left(x_{k}\right)-A(x), a(y)-a\left(x_{k}\right)\right)\right| \leq\left\|A\left(x_{k}\right)-A(x)\right\|\left\|a(y)-a\left(x_{k}\right)\right\| .
$$

Since $a: K \rightarrow X$ is weak to weak continuous and $K$ is weakly compact, $a\left(x_{k}\right)-a(y)$ is bounded. Let $M>0$ such that

$$
\left\|a\left(x_{k}\right)-a(x)\right\| \leq M, \quad \forall k \in N
$$

Therefore

$$
\left|\left\langle A\left(x_{k}\right)-A(x), a(y)-a\left(x_{k}\right)\right\rangle\right| \leq M\left\|A\left(x_{k}\right)-A(x)\right\|, \quad \forall y \in K .
$$

Since $A$ is weak to norm sequentially continuous we have

$$
\left\langle A\left(x_{k}\right)-A(x), a(y)-a\left(x_{k}\right)\right\rangle \rightarrow 0, \quad k \rightarrow \infty .
$$

Therefore, for all $y \in K, k \rightarrow \infty$, we have

$$
\left\langle A\left(x_{k}\right)-A(x), a(y)-a\left(x_{k}\right)\right\rangle \rightarrow\langle A(x), a(y)-a(x)\rangle \notin-\operatorname{int} C(x),
$$

and from

$$
\left\langle A\left(x_{k}\right), a(y)-a\left(x_{k}\right)\right\rangle \notin-\operatorname{int} C(x)
$$

we have

$$
\left\langle A(x), a(y)-a\left(x_{k}\right)\right\rangle \notin-\operatorname{int} C(x) .
$$

Hence $x \in G(y)$, which shows that $G(y)$ is weakly sequentially closed for all $y \in K$. 
Now we prove that $G(y)$ is weakly compact for all $y \in K$. In fact, let $\left\{x_{n}\right\} \subseteq G(y)$. Since $G(y) \subseteq K$ and $\left\{x_{n}\right\} \subseteq K$. Again since $K$ is weakly compact and from the Eberlein-Smulian theorem [26], $K$ is weakly sequentially compact. Hence there exists a subsequence $\left\{x_{n_{k}}\right\}$ of $\left\{x_{n}\right\}$ converges weakly to $x \in K$. Then $\left\{x_{n_{k}}\right\} \subseteq G(y)$ and from weak sequentially closedness of $G(y)$, we have $x \in G(y)$. Hence $G(y)$ is weakly sequentially compact and from the Eberlein-Smulian theorem it is weakly compact. Therefore $G(y)$ is weakly compact and weakly closed for all $y \in K$. Let $y_{1}, \ldots, y_{n} \in K$ and $y \in \operatorname{co}\left\{y_{1}, \ldots, y_{n}\right\}$. We show that

$$
y \in \bigcup_{i=1}^{n} G\left(y_{i}\right)
$$

Suppose to the contrary that $y \notin G\left(y_{i}\right)$ for every $i \in\{1,2, \ldots, n\}$. Hence

$$
\left\langle A(y), a\left(y_{i}\right)-a(y)\right\rangle \in-\operatorname{int} C(x), \quad \text { for every } i \in\{1,2, \ldots, n\} .
$$

Since $a$ is quasilinear, from Theorem 2.18 we have

$$
a(y) \in \operatorname{co}\left\{a\left(y_{1}\right), a\left(y_{2}\right), \ldots, a\left(y_{n}\right)\right\}
$$

Hence

$$
a(y)=\sum_{i=1}^{n} \lambda_{i} a\left(y_{i}\right) \quad \text { with } \lambda_{i} \geq 0, i \in\{1, \ldots, n\}, \sum_{i=1}^{n} \lambda_{i}=1 .
$$

From (5) we have

$$
\left\langle A(y), \sum_{i=1}^{n} \lambda_{i} a\left(y_{i}\right)-a(y)\right\rangle \in-\operatorname{int} C(x),
$$

a contradiction.

Hence $G$ is a KKM mapping and it satisfies all the conditions in Ky Fan's lemma. Consequently

$$
\bigcap_{y \in K} G(y) \neq \emptyset
$$

Hence the generalized vector variational inequality (1) admits a solution.

Corollary 3.6 Assume that $K$ is a weakly compact convex subset of $X$ and $X^{*}$ its topological dual. Let $A: K \rightarrow X^{*}$ be a single-valued operator. Assume that $C: K \rightarrow 2^{X}$ is a mapping with closed convex solid cone values and for each $x \in K$, int $C(x) \neq \emptyset$. If $A$ is weak to $\|\cdot\|$-sequentially continuous, then (2) admits a solution.

Theorem 3.7 Let $X$ be a reflexive Banach space, $X^{*}$ be the topological dual of $X^{*}$, and $K$ be a convex and closed subset of $X$. Let $A: K \rightarrow X^{*}$ and $a: K \rightarrow X$ be given operators. Assume that $C: K \rightarrow 2^{X}$ is a mapping with closed convex solid cone values and for each 
$x \in K$, int $C(x) \neq \emptyset$. If $A$ is weak to $\|\cdot\|$-sequentially continuous and $a$ is quasilinear and weak to weak sequentially continuous, then there exists $y_{0} \in K$ such that

$$
\lim \inf _{\|x\| \rightarrow \infty, x \in K}\left\langle A(x), a(x)-a\left(y_{0}\right)\right\rangle \in-\operatorname{int} C(x) .
$$

Then the generalized vector variational inequality (1) admits a solutions.

Proof Define a mapping $G: K \rightarrow 2^{K}$ by

$$
G(y)=\{x \in K:\langle A(x), a(y)-a(x)| \notin-\operatorname{int} C(x)\}, \quad \forall y \in K .
$$

It suffices to prove $G(y)$ is closed for all $y \in K$ and $G\left(y_{0}\right)$ is weakly compact. The rest is similar to the proof given in Theorem 3.5. In the same way as in the proof of Theorem 3.5 we can prove that $G$ is weakly sequentially closed for all $y \in K$. Since $G(y)$ is sequentially closed in the norm topology for all $y \in K, G(y)$ is closed for all $y \in K$.

Next we show that $G\left(y_{0}\right)$ is bounded. Suppose to the contrary that there exists $\left\{x_{k}\right\} \subseteq$ $G\left(y_{0}\right)$ such that $\left\|x_{k}\right\| \rightarrow \infty, k \rightarrow \infty$. Since $x_{k} \in G\left(y_{0}\right)$ for all $k \in N$ we have

$$
\left\langle A\left(x_{k}\right), a\left(x_{k}\right)-a\left(y_{0}\right)\right\rangle \notin-\operatorname{int} C(x), \quad k \in N,
$$

hence

$$
\lim \inf _{\left\|x_{k}\right\| \rightarrow \infty}\left\langle A\left(x_{k}\right), a\left(x_{k}\right)-a\left(y_{0}\right)\right\rangle \notin-\operatorname{int} C(x) .
$$

It is a contradiction. Hence $G\left(y_{0}\right)$ is bounded and weakly sequentially closed. Therefore there exists $N>0$ such that $G\left(y_{0}\right) \subseteq B_{N}$ where $B_{N}$ is the closed ball centered in 0 with radius $N$. $X$ is reflexive and $B_{N}$ is weakly compact. It follows from the proof of Theorem 3.5 that $G\left(y_{0}\right)$ is weakly compact.

Theorem 3.8 Let $K$ be a weakly compact convex subset of a Banach space $X$ and $X^{*}$ be the topological dual of $X$. Let $A: K \rightarrow X^{*}$ and $a: K \rightarrow X$ be two given operators. Assume that $C: K \rightarrow 2^{X}$ is a mapping with closed convex solid cone values and for each $x \in K$, int $C(x) \neq \emptyset$. Assume that the following conditions are fulfilled:

(a) $a$ is quasilinear,

(b) if $\left\{x_{n}\right\} \subseteq K$ converges weakly to $x \in K$ then

$$
\lim \inf _{n \rightarrow \infty}\left\langle A\left(x_{n}\right), y\right\rangle \leq\langle A(x), y\rangle \notin-\operatorname{int} C(x), \quad \forall y \in K,
$$

(c) the function $x \mapsto\langle A(x), a(x)\rangle: K \rightarrow X$ is sequentially weakly lower semi-continuous.

Then generalized vector variational inequality (1) admits a solution.

Proof Define a mapping $G: K \rightarrow 2^{K}$ by

$$
G(y)=\{x \in K:\langle A(x), a(y)-a(x)\rangle \notin-\operatorname{int} C(x)\}, \quad \forall y \in K .
$$

It suffices to show that $G(y)$ is weakly sequentially closed for all $y \in K$. The rest is similar to the proof given in Theorem 3.5 and we will omit. For all $y \in K, G(y) \neq \emptyset$ and $y \in G(y)$. For 
$y \in K$ consider a sequence $\left\{x_{k}\right\} \subseteq G(y)$ converging weakly to $x \in K$. We prove that $x \in G(y)$.

Since

$$
\begin{aligned}
& \left\langle A\left(x_{k}\right), a(y)-a\left(x_{k}\right)\right\rangle \notin-\operatorname{int} C(x), \\
& \left\langle A\left(x_{k}\right), a\left(x_{k}\right)\right\rangle \leq\left\langle A\left(x_{k}\right), a(y)\right\rangle \notin-\operatorname{int} C(x) .
\end{aligned}
$$

Since the function

$$
x \mapsto\langle A(x), a(y)\rangle: K \rightarrow X
$$

is sequentially weakly lower semi-continuous, we have

$$
\langle A(x), a(x)\rangle \leq \lim \inf _{k \rightarrow \infty}\left\langle A\left(x_{k}\right), a\left(x_{k}\right)\right\rangle \leq \lim \inf _{k \rightarrow \infty}\left\langle A\left(x_{k}\right), a(y)\right\rangle \notin-\operatorname{int} C(x) .
$$

On the other hand from (b) we have

$$
\lim \inf _{k \rightarrow \infty}\left\langle A\left(x_{k}\right), a(y)\right\rangle \leq\langle A(x), a(y)\rangle \notin-\operatorname{int} C(x)
$$

and

$$
\langle A(x), a(x)\rangle \leq\langle A(x), a(y)\rangle \notin-\operatorname{int} C(x),
$$

which shows that $x \in G(y)$. Hence $G(y)$ is weakly sequentially closed for all $y \in K$.

Theorem 3.9 Let $X$ be a reflexive Banach space, $X^{*}$ is the topological dual of $X$ and $K \subseteq$ $X$ be a closed and convex subset. Let $A: K \rightarrow X^{*}$ and $a: K \rightarrow X$ be the given operators. Assume that $C: K \rightarrow 2^{X}$ is a mapping with closed convex solid cone values and for each $x \in K$, int $C(x) \neq \emptyset$. If the following conditions are fulfilled:

(a) $a$ is quasilinear,

(b) if $\left\{x_{n}\right\} \subseteq K$ converges weakly to $x \in K$ then

$$
\lim \inf _{n \rightarrow \infty}\left\langle A\left(x_{n}\right), y\right\rangle \leq\langle A(x), y\rangle \notin-\operatorname{int} C(x), \quad \forall y \in K,
$$

(c) the function $x \mapsto\langle A(x), a(x)\rangle: K \rightarrow X$ is sequentially weakly lower semi-continuous,

(d) there exists $y_{0} \in K$ such that

$$
\lim \inf _{\|x\| \rightarrow \infty, x \in K}\left\langle A(x), a(x)-a\left(y_{0}\right)\right\rangle \in-\operatorname{int} C(x) .
$$

Then the generalized vector variational inequality (1) admits a solution.

Proof In a similar way, we can prove that $G(y)$ (see Theorem 3.8 ) is weakly sequentially closed for all $y \in K$. From Theorem 3.7, $G(y)$ is closed for all $y \in K$ and $G\left(y_{0}\right)$ is weakly compact. The rest of the proof is similar to the proof of Theorem 3.5.

Definition 3.10 Let $A: D \subseteq X \rightarrow X^{*}$ be an operator. We say that $A$ is monotone (in the Minty-Browder sense) [27] if for all $x, y \in D$ we have

$$
\langle A(x)-A(y), x-y\rangle \notin-\operatorname{int} C(x) .
$$


Remark 3.11 If operator $A: K \rightarrow X^{*}$ is hemi-continuous and monotone in the MintyBrowder sense then the solutions of the Minty generalized vector variational inequality (3) and the Minty general vector variational inequality (4) coincide.

Theorem 3.12 Let $X$ be a reflexive Banach space, $K \subseteq X$ be a closed and convex subset, and $X^{*}$ be the topological dual of $X$. Let $A: K \rightarrow X^{*}, a: K \rightarrow X$ be the given operators. Assume that $C: K \rightarrow 2^{X}$ is a mapping with closed convex solid cone values and for each $x \in K$, int $C(x) \neq \emptyset$. Then the following conclusions hold:

(i) If $A: K \rightarrow K$ is hemi-continuous and $K$ is convex, then every solution $x \in K$ of (4) is also a solutions of general vector variational inequality (2).

(ii) If $A$ is monotone on the convex set $K$, then every solution $x \in K$ of the Minty generalized vector variational inequality (2) is also a solution of the Minty general vector variational inequality (4).

Definition 3.13 Let $X$ be a Banach space, $X^{*}$ be the topological dual of $X$. Let $A: D \subseteq$ $X \rightarrow X^{*}$ and $a: D \rightarrow X$ be given operators. We say that $A$ is monotone with respect to $a$, if for all $x, y \in D$ we have

$$
\langle A(x)-A(y), a(x)-a(y)\rangle \notin-\operatorname{int} C(x) .
$$

We say that $A$ is $a$-pseudomonotone if

$$
\langle A(x), a(y)-a(x)\rangle \notin-\operatorname{int} C(x)
$$

implies

$$
\langle A(y), a(y)-a(x)\rangle \notin-\operatorname{int} C(x), \quad \forall x, y \in D .
$$

Remark 3.14 If $a \equiv \mathrm{id}_{D}$ we obtain the definition of Minty-Browder monotonicity and pseudomonotonicity, respectively. It is well known $[27,28]$ that monotonicity implies pseudomonotonicity but the converse is not true.

Theorem 3.15 Let $X$ be a reflexive Banach space, $X^{*}$ its topological dual of $X$ and $K \subseteq X$ be a closed and convex subset. Let $A: K \rightarrow X^{*}, a: K \rightarrow X$ be the given operators. Assume that $C: K \rightarrow 2^{X}$ is a mapping with closed convex solid cone values and, for each $x \in K$, int $C(x) \neq \emptyset$. Then the following conclusions hold:

(i) If $A: K \rightarrow K$ is monotone with respect to a, then every solution $x \in K$ of generalized vector variational inequality (1) is also a solution of the Minty generalized vector variational inequality (3).

(ii) If $A$ is hemi-continuous and $a$ is strictly quasilinear then every solution $x \in K$ of the Minty generalized vector variational inequality (3) is also a solution of generalized vector variational inequality (1).

Proof (i) Let $x \in K$ be a solution of (1), then

$$
\langle A(x), a(y)-a(x)\rangle \notin-\operatorname{int} C(x), \quad \forall y \in K .
$$


On the other hand, since $A$ is monotone with respect to $a$, we have

$$
\langle A(y)-A(x), a(y)-a(x)\rangle \notin-\operatorname{int} C(x), \quad \forall x, y \in K
$$

hence

$$
\langle A(y), a(y)-a(x)| \geq\langle A(x), a(y)-a(x)\rangle \notin-\operatorname{int} C(x), \quad \forall x, y \in K
$$

and

$$
\langle A(y), a(y)-a(x)\rangle \notin-\operatorname{int} C(x), \quad \forall x, y \in K .
$$

(ii) Let $x \in K$ be a solution of the Minty generalized vector variational inequality (3). Then

$$
\langle A(y), a(y)-a(x)\rangle \notin-\operatorname{int} C(x), \quad \forall y \in K
$$

Let $z \in K, z \neq x$. Since $K$ is convex we have

$$
x+t(z-x) \in K, \quad \forall t \in[0,1] .
$$

Since $a$ is strict quasilinear,

$$
\begin{aligned}
& a(x+t(z-x)) \in(a(x), a(z)), \quad \forall t \in(0,1), \\
& a(x+t(z-x))=a(x)+\alpha(a(z)-a(x)), \quad \text { for some } \alpha \in(0,1) .
\end{aligned}
$$

Consequentially we have

$$
\langle A(x+t(z-x)), a(x+t(z-x))-a(x)\rangle \notin-\operatorname{int} C(x), \quad \forall x, y \in K,
$$

or equivalently

$$
\langle A(x+t(z-x)), \alpha(a(z)-a(x))\rangle \notin-\operatorname{int} C(x), \quad \forall x, y \in K .
$$

Letting $t \downarrow 0$, taking the limit, and noting that $\alpha$ and $A$ are hemi-continuous, we have

$$
\langle A(x), a(z)-a(x)\rangle \notin-\operatorname{int} C(x), \quad \forall x, y \in K .
$$

Since $z \in K$ is arbitrary, we have

$$
\langle A(x), a(z)-a(x)\rangle \notin-\operatorname{int} C(x), \quad \forall z \in K .
$$

Hence $x$ is a solution of generalized vector variational inequality (1). 
Authors' contributions

All authors read and approved the final manuscript.

\section{Author details}

${ }^{1}$ Center for General Education, China Medical University, Taichung, 40402, Taiwan. ${ }^{2}$ Department of Mathematics, Jazan University, Jazan, Kingdom of Saudi Arabia. ${ }^{3}$ College of Statistics and Mathematics, Yunnan University of Finance and Economics, Kunming, Yunnan, 650221, P.R. China.

\section{Acknowledgements}

The authors would like to express their thanks to the editors and the referees for their kind and helpful comments and advices. This work was supported by the National Natural Science Foundation of China (Grant No. 11361070).

Received: 6 April 2015 Accepted: 5 October 2015 Published online: 21 November 2015

\section{References}

1. Stampacchia, G: Formes bilinéaires coercitives sur les ensembles convexes. C. R. Acad. Sci. Paris, Ser I. Math. 258 4413-4416 (1964)

2. Giannessi, F: Theorems of alternative, quadratic programs and complementarity problems. In: Cottle, RW, Giannessi, F, Lions, JL (eds.) Variational Inequalities and Complementarity Problems, pp. 151-186. Wiley, Chichester (1980)

3. Bensoussan, A, Lions, JL: Applications des Inéquations Variationnelles en Contrôle Stochastique. Dunod, Paris (1978)

4. Chang, SS, Zhang, Y: Generalized KKM theorem and variational inequalities. J. Math. Anal. Appl. 159, 208-223 (1991)

5. Giannessi, F: Vector Variational Inequalities and Vector Equilibrium. Kluwer Academic, Dordrecht (2000)

6. Hyers, DH: On the stability of the linear functional equation. Proc. Natl. Acad. Sci. USA 27, $222-224$ (1941)

7. Kassay, G, Pinta, C, Szenkovits, F: On convexity of preimages of monotone operators. Taiwan. J. Math. 13, 675-686 (2009)

8. Maugeri, A, Raciti, F: On existence theorems for monotone and nonmonotone variational inequalities. J. Convex Anal. $16,899-911$ (2009)

9. Székelyhidi, L: On a theorem of Baker, Lawrence and Zorzitto. Proc. Am. Math. Soc. 84, 95-96 (1982)

10. Ulam, SM: Problems in Modern Mathematics. Science Editions. Wiley, New York (1960)

11. Ferrentino, R: Variational inequalities and optimization problems. Appl. Math. Sci. 1, 2327-2343 (2007)

12. Goreham, A: Sequential convergence in topological spaces. arXiv:math/0412558

13. Khan, MF, Salahuddin: On generalized vector variational like inequalities. Nonlinear Anal. 59, $879-889$ (2004)

14. Kien, BT, Wong, NC, Yao, JC: Generalized vector variational inequalities with star pseudo monotone and discontinuous operators. Nonlinear Anal. 68, 2859-2871 (2008)

15. Lee, BS, Khan, MF, Salahuddin: Hybrid type set valued variational like inequalities in reflexive Banach spaces. J. Appl. Math. Inform. 27(5-6), 1371-1379 (2009)

16. Lee, BS, Lee, GM: Vector variational type inequalities for set valued mappings. Appl. Math. Lett. 13, 57-62 (2000)

17. Lin, LJ, Chuang, CS, Yu, ZT: Generalized KKM theorems and common fixed point theorems. Nonlinear Anal. 74(16), 5591-5599 (2011)

18. Minty, GJ: Monotone (nonlinear) operators in Hilbert spaces. Duke Math. J. 29, 341-346 (1962)

19. Salahuddin, Ahmad, MK, Verma, RU: Existence solutions to mixed vector variational type inequalities. Adv. Nonlinear Var. Inequal. 16(1), 115-123 (2013)

20. Yao, JC: General variational inequalities in Banach spaces. Appl. Math. Lett. 5, 51-54 (1992)

21. Zeng, LC, Wu, SY, Yao, JC: Generalized KKM theorem with applications to generalized minimax inequalities and generalized equilibrium problems. Taiwan. J. Math. 10(6), 1497-1514 (2006)

22. Laszlo, S: Some existence results of solutions for general variational inequalities. J. Optim. Theory Appl. 150, 425-443 (2011)

23. Laszlo, S: Generalized monotone operators, generalized convex functions and closed countable sets. J. Convex Anal. 18, 1075-1091 (2011)

24. Avriel, M, Diewert, WT, Schaible, S, Zang, I: Generalized Concavity. Plenum, New York (1988)

25. Fan, K: A generalization of Tychonoff's fixed point theorems. Math. Ann. 142, 305-310 (1961)

26. Fabian, M, Habala, P, Hajek, P, Monlesinos Santalucia, V, Lelant, J, Zizler, V: Variational Analysis and Infinite Dimensional Geometry. Springer, New York (2001)

27. Giannessi, F, Maugeri, A: Variational Inequalities and Network Equilibrium Problems. Plenum, New York (1995)

28. Chen, GY: Existence of solutions for a vector variational inequalities: an extension of Hartman-Stampacchia theorems J. Optim. Theory Appl. 74(3), 445-456 (1992) 